\title{
Erratum to: The Preventive Effects of Posterior Pericardiotomy with Intrapericardial Tube on the Development of Pericardial Effusion, Atrial Fibrillation, and Acute Kidney Injury after Coronary Artery Surgery: A Prospective, Randomized, Controlled Trial
}

\section{Mehmet Kaya ${ }^{1}$ Ayfer Utku Savaş ${ }^{2}$ Korhan Erkanli ${ }^{1} \quad$ Salih Güler $^{1} \quad$ Mugisha Kyaruzi $^{1}$ Ali Birant ${ }^{3}$} Mehmet Karaçalilar ${ }^{1}$ Murat Akkuş ${ }^{4}$ Ihsan Bakir ${ }^{1}$

\footnotetext{
1 Department of Cardiovascular Surgery, Istanbul Mehmet Akif Ersoy Thoracic and Cardiovascular Surgery Training and Research Hospital, Istanbul, Turkey

2 Department of Pulmonology, Istanbul Mehmet Akif Ersoy Thoracic and Cardiovascular Surgery Training and Research Hospital, Istanbul, Turkey 3 Department of Cardiology, Istanbul Mehmet Akif Ersoy Thoracic and Cardiovascular Surgery Training and Research Hospital, Istanbul, Turkey ${ }^{4}$ Department of Thoracic Surgery, Istanbul Mehmet Akif Ersoy Thoracic and Cardiovascular Surgery Training and Research Hospital, Istanbul, Turkey
}

Address for correspondence Mehmet Kaya, MD, Department of Cardiovascular Surgery, Istanbul Mehmet Akif Ersoy Thoracic and Cardiovascular Surgery Training and Research Hospital, Mehmet Akif Ersoy GKDC Eğitim Hastanesi Halkali Küçükçekmece, Istanbul 34000, Turkey (e-mail: drmehmetkaya@yahoo.com).

Thorac Cardiovasc Surg 2016;64:e1-e2.

ERRATUM

It has been brought to the Editor's attention that Table 2 in the above publication contains an error in measurement units. The amount of pericardial effusion by echocardiography is measured in mm, not in $\mathrm{mL}$. Article published eFirst on April 14, 2015. DOI of the original article: $10.1055 / \mathrm{s}-0035-1548737$.

After consultation with the authors the corrected and re-arranged table appears as follows:
Markus K. Heinemann, MD, PhD Editor-in-Chief The Thoracic and Cardiovascular Surgeon 
Table 2 Surgical and postoperative data

\begin{tabular}{|c|c|c|c|}
\hline & $\begin{array}{l}\text { Control group } \\
(n=107) \\
([\text { mean } \pm \text { SD]; }[n, \%])\end{array}$ & $\begin{array}{l}\text { Study group } \\
(n=103) \\
([\text { mean } \pm S D] ;[n, \%])\end{array}$ & $p$ Value \\
\hline \multicolumn{4}{|l|}{ Intraoperative variables } \\
\hline CPB time (min) & $77.02 \pm 22.83$ & $81.6 \pm 26.53$ & 0.213 \\
\hline Cross-clamp time (min) & $42.89 \pm 14.91$ & $45.47 \pm 19.05$ & 0.313 \\
\hline Number of distal anastomoses & $2.88 \pm 0.85$ & $3.01 \pm 1.08$ & 0.336 \\
\hline \multicolumn{4}{|l|}{ AKI } \\
\hline Postoperative day 2 & $23(21.50 \%)$ & $17(16.50 \%)$ & 0.357 \\
\hline Postoperative day 5 & $18(16.82 \%)$ & $4(3.92 \%)$ & 0.002 \\
\hline Postoperative day 20 & $20(18.69 \%)$ & $7(6.86 \%)$ & 0.011 \\
\hline New postoperative dialysis (transient) & $1(0.93 \%)$ & 0 & 0.328 \\
\hline $\mathrm{s} \mathrm{Cr}$ level on the postoperative day $2(\mathrm{mg} / \mathrm{dL})$ & $0.95 \pm 0.25$ & $0.89 \pm 0.23$ & 0.085 \\
\hline $\mathrm{s} C r$ level on the postoperative day $5(\mathrm{mg} / \mathrm{dL})$ & $0.87 \pm 0.24$ & $0.86 \pm 0.35$ & 0.738 \\
\hline $\mathrm{sCr}$ level on the postoperative day $20(\mathrm{mg} / \mathrm{dL})$ & $0.9 \pm 0.37$ & $0.83 \pm 0.21$ & 0.116 \\
\hline$p$ (comparison of intragroup for $\mathrm{s} C r$ ) & 0.001 & 0.026 & \\
\hline eGFR value on the postoperative day $2(\mathrm{~mL} / \mathrm{min})$ & $99.73 \pm 39.76$ & $105.56 \pm 33.10$ & 0.250 \\
\hline eGFR value on the postoperative day $5(\mathrm{~mL} / \mathrm{min})$ & $110.1 \pm 46.60$ & $113.06 \pm 34.92$ & 0.604 \\
\hline eGFR value on the postoperative day $20(\mathrm{~mL} / \mathrm{min})$ & $106.55 \pm 37.65$ & $112.08 \pm 30.55$ & 0.245 \\
\hline$p$ (comparison of intragroup for eGFR) & 0.0001 & 0.001 & \\
\hline \multicolumn{4}{|c|}{ Amount of pericardial effusion by echocardiography $(\mathrm{mm})$} \\
\hline Postoperative day 2 & $2.42 \pm 5.29$ & $1.17 \pm 2.7$ & 0.034 \\
\hline Postoperative day 5 & $2.5 \pm 5.02$ & $1.15 \pm 2.92$ & 0.019 \\
\hline Postoperative day 20 & $1.89 \pm 4.50$ & $0.93 \pm 5.62$ & 0.174 \\
\hline Amount of the total drainage $(\mathrm{mL})$ & $531.86 \pm 289.83$ & $562.76 \pm 365.19$ & 0.589 \\
\hline Amount of mediastinal drainage & $205.41 \pm 154.51$ & $270.49 \pm 225.90$ & 0.058 \\
\hline Amount of pleural drainage & $299 \pm 185.11$ & $281.67 \pm 215.69$ & 0.616 \\
\hline \multicolumn{4}{|l|}{ Complications/morbidity } \\
\hline Bleeding-related revision & $2(1.87 \%)$ & $1(0.97 \%)$ & 0.589 \\
\hline Tamponade & $4(3.74 \%)$ & 0 & 0.048 \\
\hline Packed red blood cells (units) & $2.07 \pm 1.69$ & $2.28 \pm 2.13$ & 0.465 \\
\hline POAF & $30(28.04 \%)$ & $15(14.56 \%)$ & 0.019 \\
\hline Postoperative EF (5) & $52.69 \pm 9.41$ & $53.29 \pm 7.43$ & 0.704 \\
\hline Cardioversion & $1(0.93 \%)$ & 0 & 0.328 \\
\hline Mechanical ventilation time $(\mathrm{h})$ & $6.8 \pm 2.46$ & $6.46 \pm 2.06$ & 0.298 \\
\hline Reintubation & $1(0.93 \%)$ & 0 & 0.328 \\
\hline Atelectasis & $7(6.54 \%)$ & $2(1.94 \%)$ & 0.103 \\
\hline Intervention to the pleural effusion & $9(8.41 \%)$ & $6(5.83 \%)$ & 0.479 \\
\hline Postoperative CVA & 0 & 0 & - \\
\hline Intra-aortic balloon pump & $1(0.93 \%)$ & 0 & 0.328 \\
\hline Length of stay (d) & $7.33 \pm 4.05$ & $6.11 \pm 2.31$ & 0.009 \\
\hline Rehospitalization & $7(6.54 \%)$ & $4(3.88 \%)$ & 0.396 \\
\hline Perioperative inotrope & $11(10.28 \%)$ & $8(7.77 \%)$ & 0.540 \\
\hline Mortality & $1(0.93 \%)$ & 0 & 0.328 \\
\hline
\end{tabular}

Abbreviations: AKI, acute kidney injury; CPB, cardiopulmonary bypass; CVA, cerebrovascular accident; eGFR, estimated glomerular filtration rate; POAF, postoperative atrial fibrillation; $\mathrm{sCr}$, serum creatinine. 